\title{
Second salvage treatment for local recurrence of prostate cancer using high-dose-rate brachytherapy: a case report
}

\author{
Audrey Claren, MD', Mathieu Gautier, MSc², Julien Feuillade, MSc², Alexander Tuan Falk, MD, PhD?, \\ Jean-Michel Hannoun Levi, MD, PhD' \\ 'Unit of Radiation Oncology, ${ }^{2}$ Unit of Radiation Physics, Centre Antoine Lacassagne, University of Nice-Sophia, Nice, France
}

\begin{abstract}
Purpose: Currently, there are no recommendations for the management of a second local recurrence of prostate adenocarcinoma except for the introduction of androgen deprivation therapy (ADT).

Case report: A 69-year-old man underwent a third salvage local treatment with high-dose-rate brachytherapy (HDRB), for a second biochemical relapse for local recurrence. Thirty-five Grays in 5 fractions were delivered on the whole prostate extended to the proximal part of left seminal vesicle. Given the availability of new treatment techniques in our radiation therapy department, a dosimetric comparison between HDRB and stereoatactic radiosurgery (SRS) was performed.

Results: Immediate tolerance of HDRB was acceptable with achievement of prostate specific antigen (PSA) nadir in 24 months $(0.03 \mathrm{ng} / \mathrm{ml})$. Observed late toxicities were only grade 2 urinary incontinence. Dosimetric comparison showed a slight advantage on clinical target volume coverage and rectum protection for the SRS. The HDRB showed an advantage on bone irradiation including femoral heads and the volume receiving $0.5 \mathrm{~Gy}(\mathrm{EQD} 2=1 \mathrm{~Gy}$ with $\alpha / \beta=3)$.

Conclusions: A third local treatment with good tolerance could be a therapeutic option in case of a second local prostate cancer recurrence in order to delay, as long as possible, the chemical castration. Both techniques (HDRB and SRS) seem valid and should be chosen based on the availability and experience in a treatment center.
\end{abstract}

Key words: brachytherapy, prostate cancer, recurrence, stereoatactic radiosurgery.

\section{Purpose}

Currently, there are no recommendations for the management of a second local recurrence of prostate adenocarcinoma except for the introduction of palliative androgen deprivation therapy (ADT). Improved diagnostic techniques including functional magnetic resonance imaging (MRI) and positron emission tomography imaging (PET) [1] with choline derived tracers enables to more accurately detect the site of recurrence in case of subsequent biochemical relapse. The development of new therapeutic approaches, including local treatment, allows to consider other strategies. Androgen deprivation therapy can often be poorly tolerated (psychologic, metabolic, cardio-vascular and bone complications), with an unclear impact on specific survival [2]; it could be delayed for a selected group of patients. Two radiotherapy techniques have already been investigated in the context of first salvage treatment for prostate cancer local recurrence: brachytherapy (low- or high-dose-rate) and stereoatactic radiosurgery (SRS) [3].

\section{Case report}

\section{Patient presentation}

We report the case of a 60-year-old man who underwent high-dose-rate brachytherapy (HDRB) as a salvage treatment for second biochemical relapse due to second local recurrence. In November 1997, he presented an intermediate-risk prostate cancer (histological details not available). He underwent an external beam radiation therapy delivering a total dose of $70 \mathrm{~Gy}$ in 35 fractions combined with a 6 months ADT.

In 2006, prostate specific antigen (PSA) level increased to $3.87 \mathrm{ng} / \mathrm{ml}$. While computed tomography (CT) scan and bone scan were considered as normal, transperineal ultrasound-guided prostatic biopsies confirmed a Gleason score $9(4+5)$ local recurrence (all the left lobe and the right median apex). A local treatment by high-intensity focused ultrasound (HIFU) was performed, which led to a PSA decrease to $0.04 \mathrm{ng} / \mathrm{ml}$.

In 2011, due to rising PSA $(3.84 \mathrm{ng} / \mathrm{ml})$, extension work-up based on CT-scan, MRI, and bone scan was per-
Address for correspondence: Audrey Claren, MD, Radiation Therapy Department, Centre Antoine Lacassagne, 33 Avenue Valombrose, 06189 Nice CEDEX 2, France, phone: +33 618077040, fax: +33 492031574, a e-mail: audreyclaren@hotmail.fr
Received: 13.03 .2015

Accepted: 14.04 .2015

Published: 25.06.2015 
formed to exclude the possibility of distant metastases. Furthermore, MRI confirmed the involvement of the left seminal vesicle. New biopsies were carried out confirming second local recurrence, Gleason score $10(5+5)$. A salvage HDRB was proposed as a treatment and patient was informed about the risks of urinary and rectal side effects. An institutional multidisciplinary expert committee confirmed this therapeutic option, and a signed-consent form was obtained.

\section{Treatment modalities}

Brachytherapy was performed under general anaesthesia after urinary catheterization. Using trans-rectal ultrasound (TRUS) guidance, 10 catheters (Sharp Needles ${ }^{\mathrm{TM}}$, Elekta AB, Stockholm, Sweden) were implanted trans-perineally and peri-urethrally through a dedicated template (Fig. 1). Dosimetric CT-scans were performed daily, before each fraction, followed by re-optimization in regards to the risk of catheter migration. Clinical target volume (CTV) consisted in the whole prostate (prostatic volume $5 \mathrm{cc}$, due to a previous HIFU treatment) extended to the proximal part of left seminal vesicle. Urethra and rectum were considered as organs at risk (OARs) and were delineated. Brachytherapy delivered a total dose of $35 \mathrm{~Gy}$ in 5 fractions (7 Gy/fraction) over 5 consecutive days. The equivalent dose $\alpha / \beta=3$ at $2 \mathrm{~Gy}\left(\mathrm{EQD}_{\mathrm{\alpha} \beta 3}\right)$ was $70 \mathrm{~Gy}$ for prostate cancer. Dose constraints for OARs were: Vu115 (percentage of the urethra volume receiving $115 \%$ of the prescribed dose)

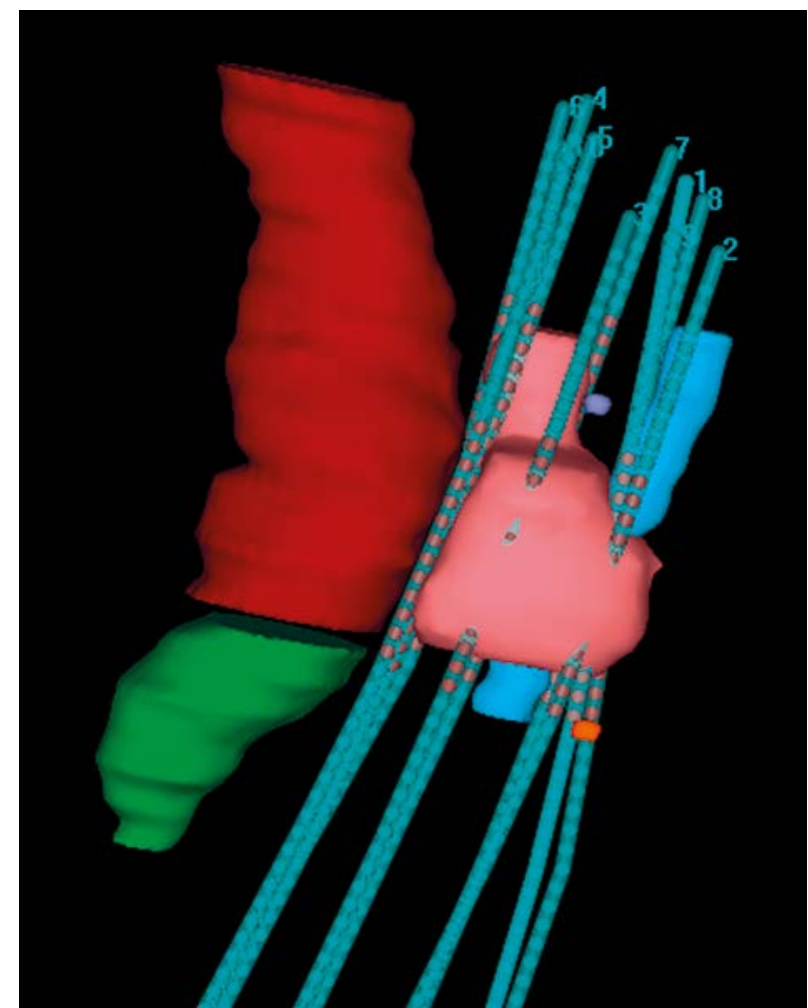

Fig. 1. Reconstruction from the dosimetric CT-scan of the brachytherapy treatment. Pink: prostate and half of left seminal vesicle. Blue: urethra. Aquamarine: 10 implanted catheters for treatment. Green: canal anal. Brown: rectum and $\mathrm{Vr}_{90}$ (percentage of the rectum volume receiving $90 \%$ of the prescribed dose). Both of them were to be less than $1 \%$ of the prescribed dose. Patient was hospitalized 6 days, from the day before the implantation to the day after the last fraction. Needles were removed just after the last fraction, under low sedation. It led to a common macroscopic haematuria managed with bladder irrigation until the bleeding stopped (24 hours). After removal of the urinary catheter and normalization of urinary function, patient was discharged.

Due to the multiplications and accessibility of new technologies, we decided to compare HDRB to SRS based on the same dosimetric CT scan, since the algorithm for calculating the SRS does not take into account the heterogeneity of volumes.

\section{Results}

\section{Clinical outcome}

Immediate tolerance was acceptable with the macroscopic hematuria managed within the 24 hours after the Folley catheter removal. One month after salvage HDBR, we noticed a nocturnal urinary frequency of 6 per night and dysuria grade 2 according to the Common Terminology Criteria for Adverse Event (CTCAE v4.0) with no grade $\geq 2$ digestive toxicities. At 24 months, late toxicities were nocturnal urinary frequency from 4 to 5 per night, urinary incontinence grade 2 with no grade $\geq 2$ digestive complication. Regarding sexual abilities, we observed an erectile dysfunction grade 3 , which was already present before treatment (the impact of salvage HDRB on sexual function has been difficult to evaluate due to a pre-therapeutic grade 3 erectile dysfunction). Efficacy at 24 months was achieved with a PSA nadir at $0.03 \mathrm{ng} / \mathrm{ml}$ without any ADT (Fig. 2).

\section{Dosimetric comparison}

Dosimetric results related to HDRB are detailed in Table 1 . The comparison of the two irradiation techniques, on the same CT-scan, was based on: percentage of the CTV receiving $100 \%$ of the dose $\left(\mathrm{CTV} \mathrm{V}_{100 \%}\right)$; dose delivered to $90 \%$ of the CTV $\left(\mathrm{CTV} \mathrm{D}_{90}\right)$; percentage of the

Table 1. High-dose-rate brachytherapy dosimetry

\begin{tabular}{lccc} 
Parameters & Mean & Minimal & Maximal \\
\hline $\mathrm{V}_{100 \%}$ & 90.31 & 89.60 & 92.58 \\
\hline $\mathrm{V}_{150 \%}$ & 27.85 & 25.43 & 34.81 \\
\hline $\mathrm{V}_{200 \%}$ & 12.60 & 11.64 & 14.78 \\
\hline $\mathrm{D}_{100}(\mathrm{~Gy})$ & 4.67 & 4.37 & 4.90 \\
\hline $\mathrm{D}_{90}(\mathrm{~Gy})$ & 7.02 & 6.96 & 7.20 \\
\hline $\mathrm{V}_{115 \%}$ urethra (cc) & 0.16 & 0.10 & 0.33 \\
\hline $\mathrm{V}_{97 \%}$ rectum (cc) & 0.04 & 0.02 & 0.05
\end{tabular}

CTV - clinical target volume; $V_{100 \%}, V_{150 \%}, V_{200 \%}, V_{115 \%}, V_{97 \%}$ - volume of the anatomic volume receiving 100\%, 150\%, 200\%, 115\%, 97\% of the prescribed dose; $D_{100}, D_{90}$ - the minimum dose to $100 \%, 90 \%$ of the CTV 


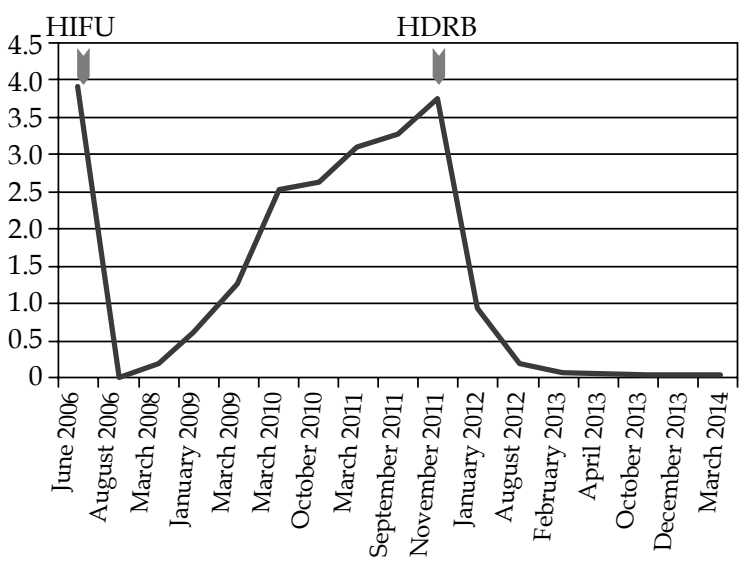

Fig. 2. Prostate specific antigen (PSA) evolution (ng/ml)

urethra volume receiving $115 \%\left(\mathrm{~V}_{115 \%}\right)$ of the prescribed dose; dose delivered to $0.1 \mathrm{cc}, 1 \mathrm{cc}$ and $2 \mathrm{cc}$ of the urethra; percentage of the rectal volume receiving $90 \%\left(\mathrm{~V}_{90 \%}\right)$ of the prescribed dose; dose delivered to $0.1 \mathrm{cc}, 1 \mathrm{cc}$ and $2 \mathrm{cc}$ the rectum; median dose delivered to right and left femoral heads and volume of the $0.5 \mathrm{~Gy}$ isodose $\left(\mathrm{EQD} 2_{\alpha / \beta 3}=\right.$ $=1 \mathrm{~Gy}$ ), intimately linked to the integral dose ([Total volume receiving $0.5 \mathrm{~Gy}$ ] - [CTV + OARs]) (Table 2 and Fig. 3).

Coverage of the target volume $\left(\mathrm{V}_{100 \%}\right.$ and $\left.\mathrm{D}_{90}\right)$ appears better with SRS $\left(\mathrm{V}_{100 \%}: 99.2 \%\right.$ vs. $90.3 \%$, and $\mathrm{D}_{90}$ : 37.5 Gy vs. $35.09 \mathrm{~Gy}$ ). Regarding the dosimetric parameters related to the OARs (urethra and rectum), the results appeared quite similar except for the rectum with higher dose with HDRB $\left(\mathrm{D}_{0.1 \mathrm{cc}}\right.$ rectum: 32.4 vs. $29.6 \mathrm{~Gy} ; \mathrm{D}_{1 \mathrm{cc}}$ rectum: 28.1 vs. $25.3 \mathrm{~Gy}$, and $\mathrm{D}_{2 \mathrm{cc}}$ rectum: 25.5 vs. $18.5 \mathrm{~Gy}$ ). Median doses delivered to right and left femoral heads were lower with HDRB (median dose: 0.15 vs. 6 Gy, 0.17 vs. 3.7 Gy for right and left femoral heads, respectively). In terms of integral dose compared to the results obtained with SRS, HDRB reduced divided the 0.5 Gy isodose volume by 2 (13 021 vs. 7200 cc).

\section{Discussion and Conclusions}

This case report highlights the possibility to perform a second salvage treatment for local recurrent prostate
Table 2. Dosimetric comparison of high-dose-rate brachytherapy (HDRB) and stereoatactic radiosurgery (SRS)

\begin{tabular}{|c|c|c|}
\hline Parameters & HDRB & SRS \\
\hline Total dose (Gy) & 35 & 35 \\
\hline Dose per fraction (Gy) & 7 & 7 \\
\hline$V_{100 \%}$ & 90.31 & 99.2 \\
\hline$D_{90}(G y)$ & 35.09 & 37.5 \\
\hline $\mathrm{V}_{115}$ urethra $(\mathrm{cc})$ & 0.16 & 0 \\
\hline $\mathrm{D}_{0.1 c c}$ urethra (Gy) & 40.72 & 39.3 \\
\hline $\mathrm{D}_{1 c c}$ urethra (Gy) & 35.9 & 37.4 \\
\hline $\mathrm{D}_{2 c c}$ urethra (Gy) & 27.1 & 18.1 \\
\hline $\mathrm{V}_{90}$ rectum $(\mathrm{cc})$ & 0.21 & 0 \\
\hline $\mathrm{D}_{0.1 \mathrm{cc}}$ rectum (Gy) & 32.4 & 29.6 \\
\hline $\mathrm{D}_{1 c c}$ rectum (Gy) & 28.1 & 25.3 \\
\hline $\mathrm{D}_{2 c c}$ rectum $(\mathrm{Gy})$ & 25.5 & 18.5 \\
\hline Median dose on right femoral head (Gy) & 0.15 & 6 \\
\hline Median dose on left femoral head (Gy) & 0.17 & 3.7 \\
\hline $\begin{array}{l}\text { Volume receiving } 0.5 \mathrm{~Gy}-[\mathrm{CTV}+\mathrm{OARs}] \\
\text { (cc) }\end{array}$ & 7200 & 13021 \\
\hline
\end{tabular}

$C T V$ - clinical target volume, $O A R$ - organs at risk, $D_{0.1 c c} D_{1 c c}, D_{2 c c}-$ minimum dose to the most exposed $0.1 \mathrm{~cm}^{3}, 1 \mathrm{~cm}^{3}, 2 \mathrm{~cm}^{3}$

cancer using HDRB. This approach could be an effective and well-tolerated medical care. The PSA nadir was reached within 24 months. As expected, the main side effect was a urinary toxicity. Fortunately, for this specific patient, no digestive toxicity was observed, however, rectal toxicity remains an important issue and could be reduced by using transperineal injection of hyaluronic acid in the anterior perirectal fat [4].

The benefit of this second salvage local treatment is to delay the beginning of ADT and its side effects (cardiovascular, osteoporosis, sexual, and psychological) [5-7].

After two salvage treatments, we noticed a rising Gleason score that could be due to either more aggressive selected cancer cells or an overestimation in correlation with the difficulty of histopathological interpretation [8].
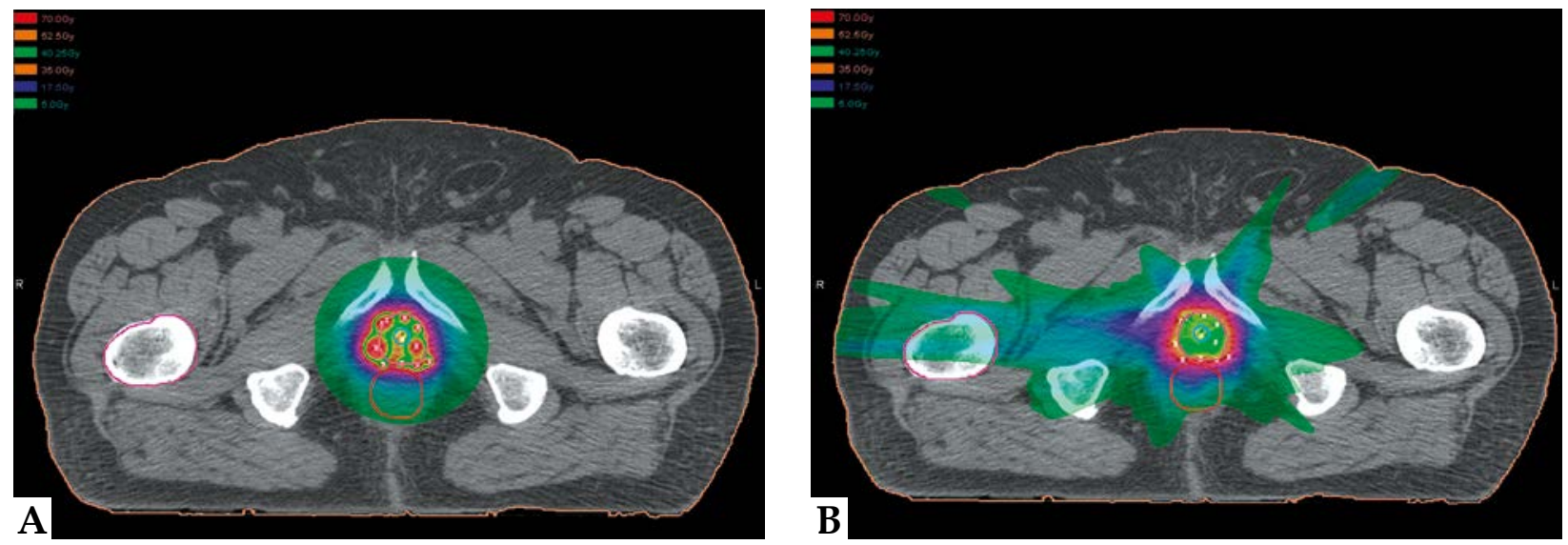

Fig. 3. Prostatic dose-distribution analysis on CT-scan axial slices for each treatment type. Prescribed dose: 35 Gy in 5 fractions. A) High-dose-rate brachytherapy. B) Stereotactic radiosurgery 
The comparison between the two techniques does not allow to conclude on the best method. The percentage of CTV receiving $100 \%$ of the prescribed dose is greater than $90 \%$ in the two techniques. Protection of OARs is respected with both techniques [9]. However, median dose to the femoral heads is higher with SRS, which has to be considered with the dose already delivered during the initial external beam radiation therapy. The most important difference highlighted related to the volume receiving $0.5 \mathrm{~Gy}\left(\mathrm{EQD} 2_{\mathrm{\alpha} \beta 3}=1 \mathrm{~Gy}\right)$, which was reduced with HDRB and seems to be correlated to the risk of radiation-induced cancers [10].

Regarding these results and imaging improvement (MRI and PET with choline derived tracers), both HDRB and SRS appear to be an attractive treatment option of localized prostate cancer recurrence. The type of treatment should be made based on the availability and experience of the techniques.

The accuracy of HDRB and SRS, and the ability to precisely define the site of prostate cancer could allow considering focal therapy for intra-prostatic small local recurrence in order to significantly reduce toxicity $[11,12]$.

\section{Acknowledgement}

Thanks to Dr Mathieu John Ouvrier for helping in translation of the manuscript.

\section{Disclosure}

Authors report no conflict of interest.

\section{References}

1. Cyran CC, Paprottka PM, Eisenblätter M et al. Visualization, imaging and new preclinical diagnostics in. Radiat Oncol 2014; 9: 3 .

2. Studer UE, Whelan $P$, Wimpissinger $F$ et al. differences in time to disease progression do not predict for cancer-specific survival in patients receiving immediate or deferred androgen-deprivation therapy for prostate cancer: final results of EORTC randomized trial 30891 with 12 years of follow-up. Eur Urol 2014; 66: 829-838.

3. Spratt DE, Scala LM, Folkert M et al. A comparative dosimetric analysis of virtual stereotactic body radiotherapy to highdose-rate monotherapy for intermediate-risk prostate cancer. Brachytherapy 2013; 12: 428-433.

4. Prada PJ, Fernández J, Martinez AA et al. Transperineal injection of hyaluronic acid in anterior perirectal fat to decrease rectal toxicity from radiation delivered with intensity modulated brachytherapy or EBRT for prostate cancer patients. Int J Radiat Oncol Biol Phys 2007; 69: 95-102.

5. Cary KC, Singla N, Cowan JE et al. Impact of androgen deprivation therapy on mental and emotional well-being in men with prostate cancer: analysis from the CaPSURE ${ }^{\mathrm{TM}}$ registry. J Urol 2014; 191: 964-970.

6. Ziehr DR, Chen M-H, Zhang D et al. Association of androgen deprivation therapy with excess cardiac-specific mortality in men with prostate cancer. BJU Int 2014; doi: 10.1111/ bju.12905.

7. Alibhai SH, Yun L, Cheung AM, Paszat L. Screening for osteoporosis in men receiving androgen deprivation therapy. JAMA 2012; 307: 255-256.
8. Molinié V, Mahjoub WK, Balaton A. Histological modifications observed in prostate after preserving treatments for prostate cancer and their impact on Gleason score interpretation. Ann Pathol 2008; 28: 363-373.

9. Timmerman RD. An Overview of Hypofractionation and Introduction to This Issue of Seminars in Radiation Oncology. Semin Radiat Oncol 2008; 18: 215-222.

10. Tukenova M, Diallo I, Hawkins $M$ et al. Long-term mortality from second malignant neoplasms in 5-year survivors of solid childhood tumors: temporal pattern of risk according to type of treatment. Cancer Epidemiol Biomark Prev 2010; 19: 707-715.

11. Sasaki H, Kido M, Miki K et al. Salvage partial brachytherapy for prostate cancer recurrence after primary brachytherapy. Int J Urol 2014; 21: 572-577.

12. Kovács G, Cosset J-M, Carey B. Focal radiotherapy as focal therapy of prostate cancer. Curr Opin Urol 2014; 24: 231-235. 